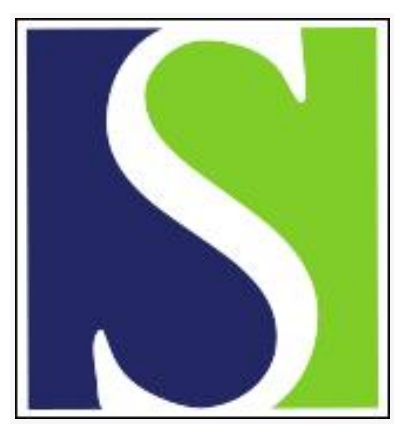

Scand J Work Environ Health 1984;10(6):481-485

https://doi.org/10.5271/sjweh.2312

Issue date: Dec 1984

\title{
Atopy and work.
}

by Nordman $\mathrm{H}$

This article in PubMed: www.ncbi.nlm.nih.gov/pubmed/6398914

\section{(c) (1)}




\title{
Atopy and work
}

\author{
Henrik Nordman, $M D^{1}$
}

\begin{abstract}
NORDMAN H. Atopy and work. Scand J Work Environ Health 10 (1984) 481-485. Atopy denotes the exceptional capacity to produce immunoglobulin (Ig)E antibody when exposed to common environmental allergens. The characteristic is frequently used for preemployment screening purposes. Too little attention has, however, been paid to the rationale and the consequences of this practice. Atopy is very common, and so decisions made because of atopy probably affect about a third of the working population. Work-related hypersensitivity symptoms cannot be eradicated by the weeding out of atopics. The intensity of exposure and/or the sensitizing properties of causative agents are often extremely strong in occupational settings and trigger the production of specific $\operatorname{IgE}$ antibodies even in nonatopics. Atopy is probably not sufficiently discriminative for screening purposes even in environments where atopics are known to have a greater risk of developing asthma (eg, laboratories with animals). Moreover, weeding out atopics may be used instead of hygienic and technical measures to reduce exposure levels. Separate decisions on medical, as well as legal, grounds may be warranted when a person with atopic symptoms, ie, rhinitis, asthma, or dermatitis, enters a new occupational environment. There is an urgent need for prospective studies in various occupational environments.
\end{abstract}

Key terms: atopy, preemployment screening, work-related symptoms.

Atopy is a term which defines a population that distinctly differs with respect to its immunologic reactivity. The term has become deeply rooted in the vocabulary of allergologists, general practitioners, and occupational health officers. However, its usefulness has been obscured by the absence of a unanimously accepted definition. Sometimes atopy is used to denote a clinical condition, sometimes an immunologic mechanism, whereas it frequently stands for a combination of both. Moreover, authors tend to forget to state clearly how the term was used in a particular context. A careless use of atopy synonymously with the far wider concept of allergy has further added to the confusion.

The predictive value of atopy has naturally attracted the interest of occupational health officers, who have used it in preemployment screening. The vagueness of the definition and the lack of data from longitudinal studies is reflected in the wide range of practices, which have mostly been unfavorable for atopics. This paper reviews and discusses the rationales for screening on the grounds of atopy and some implications of such a practice.

\section{The concept of atopy}

When the term atopy was introduced in 1923 by Coca \& Cooke (5), it was originally meant to describe individuals with a familial tendency to become sensitized.

\footnotetext{
1 Department of Occupational Medicine, Institute of Occupational Health, Helsinki, Finland.
}

Reprint requests to: $\mathrm{Dr} \mathrm{H}$ Nordman, Institute of Occupational Health, Haartmaninkatu 1, 00290 Helsinki, Finland.
It was also suggested that it be restricted to the description of human, naturally occurring hypersensitivity, manifested clinically by hay fever and asthma. Two years later, Coca \& Grove (6) launched the term "atopic reagins" for the specifically reacting substances in the serum of atopic individuals. The authors refrained from calling the substances antibodies as they considered evidence of an immunologic reaction to be lacking. The discovery of specific immunoglobulin (Ig) E antibody in 1967 confirmed that the reagins indeed were antibodies. Reagins are today synonymous with this class of antibodies, which is by no means confined to atopics or even to man (30).

The definition of atopy probably most widely accepted was offered by Pepys in 1975, ie, the capacity readily to produce IgE antibody in response to ordinary exposure to the common allergens of the subject's environment (31). This definition applies to atopy as it is used throughout the present document. Pepys further suggested that a reaction to an allergen occurring only in some occupational environments should not be called atopic, but reaginic. Thus the production of specific IgE antibody to phthalic anhydride or platinum salts, not being common environmental allergens, is not an atopic, but reaginic reaction.

Even if the definition of atopy is agreed upon, there is still a need to distinguish between atopic individuals with and without symptoms. Such differentiation is particularly important in occupational settings, where symptoms are definitely more important at the preemployment stage than atopy itself. One may talk about symptomatic and asymptomatic atopics. An expression, such as atopic predisposition, to 
define subjects who have demonstrable IgE antibodies but who have never suffered any atopic symptoms would be a valuable complement to the terminology. An individual's "atopic status" may be high or low, meaning that subjects classified as atopics with multiple sensitivity, ie, reacting to two or more allergens, have a high atopic status, whereas those with limited or isolated sensitivity have a lower status (30).

\section{The prevalence of atopy}

If a particular characteristic is used as the basis for screening, the occurrence of the feature is naturally important. Earlier studies suggested that about $10 \%$ of the population experienced some kind of atopic symptoms at some point of time (36). More recent studies support a higher prevalence of symptoms, in the range of $15-30 \%(13,15,18,25)$.

The prevalence of positive skin reactions to common environmental allergens has been studied in various "normal" populations. It is only natural that the studies show a broad range of results, as skin reactivity is dependent on a number of factors such as age, quality and number of allergen extracts, testing techniques, and, in particular, the criteria for interpreting a test as positive. Even so, the prevalence in most studies varies from 25 to $50 \%(2,9,12,17,18$, 38). It seems clear that atopy is a very common characteristic and that any action taken on the basis of it will necessarily affect a substantial proportion of the working population. It goes without saying that the practice of disqualifying job applicants because of such a common characteristic as atopy should be founded on indisputable evidence that the atopics really do run a greater risk of becoming ill in a particular environment. This evidence is lacking for the majority of occupational environments.

\section{Increased risk for atopics}

It may be anticipated that those individuals who are capable of producing IgE antibody to common allergens, eg, pollens or house-dust mite, would become sensitized more easily than others to allergens known to cause an IgE-mediated allergy. The reasoning in this line of thought seems irrefutable. A classical example would be the bakery industry; the respiratory symptoms of bakers were already described by $\mathrm{Ra}$ mazzini in 1700 (32). Although baker's asthma is a typical IgE-mediated disease (37), surprisingly little is known about both the risk of sensitization of atopics and the prognosis of sensitized workers.

According to a fairly recent study (21), comprising 234 bakers, $25 \%$ was suffering from some kind of atopic disease, and $9 \%$ was found to have asthma. Atopy was not clearly defined in this investigation, nor was it studied in the sense defined here. The authors concluded, on the premise that people who al- ready had rhinitis and/or asthma before starting work in a bakery tended to deteriorate, that atopics are unsuitable for bakery work. The prevalence of asthma was rather similar to that in another study on 242 bakers, who had a $16.5 \%$ overall prevalence of flour-induced respiratory complaints, a $7 \%$ prevalence of asthma, and $9.5 \%$ prevalence of rhinitis without concommitant asthma. Clinically healthy subjects showed prevalences of positive skin tests to house-dust mite and grass pollens of 6 and $10.5 \%$, respectively, whereas asthmatics had a higher prevalence of 29 and $65 \%$, respectively (35). These findings may be construed as an indication that atopics run a greater risk of becoming sensitized to flour dust. Both studies were cross-sectional and dealt with "survivors." They therefore tended to underestimate the risk.

Longitudinal studies on bakers are scarce. A fiveyear follow-up of bakers' apprentices showed that about $9 \%$ of newcomers may show a positive skin test to flour within a few weeks of starting work. At the end of the third year this figure had increased to $19 \%$; at this point of time complaints compatible with rhinitis or asthma were reported by $7 \%$. Without the predictive value of preexisting atopy having been specifically studied, it was noted that there was some tendency for pollen-sensitive subjects to become sensitive to flour, whereas the reverse was rare $(19,20)$.

One of the few areas in which atopy has been studied rather extensively in relation to work-related allergy is laboratory animal handling. According to some major studies, the prevalence of laboratory animal allergy is about $20 \%$, or probably a little higher, whereas the prevalence of asthma seems to be around $10 \%$ (table 1). The figures have, however, been derived from cross-sectional studies. Again one may expect atopics to be more susceptible to sensitization than nonatopics, as the animal allergies are mostly IgE-mediated. It has been suggested that atopics should not be allowed to work as laboratory animal keepers because of the alleged likelihood of developing an allergy to animals (24). Against this background the remarkably consistent results of several recent studies become interesting $(7,11,27,33)$. They show, first, that animal allergy, ie, eczema, rhinitis, or asthma, is as common among atopics as among nonatopics and, second, that atopic individuals are more inclined to progress into severe disease, ie, asthma.

Of the 179 laboratory animal workers studied by Cockcroft et al (7), 70 atopics showed a prevalence of animal allergy $(30 \%)$ similar to that of the 109 nonatopics $(26 \%)$. Atopics (ie, skin-test positives with common allergens) did have asthma more frequently (14 out of $70,20 \%$ ) than the nonatopics; however 7 cases $(6 \%)$ of asthma were also found among the 109 nonatopics. Weeding out the atopics at the preemployment examination would not have significant- 
ly decreased the overall allergy caused by contact with animals (8). However, if it is assumed that these figures show the true risk, the substitution of nonatopics for the atopics would have reduced the 21 asthmatics to 11 . This reduction would have occurred at the expense of 49 atopics who would probably not have contracted any disease, an assumption that may be wrong as the study was cross-sectional (table 2). Nevertheless, whether or not atopy can be considered sufficiently discriminative to be of use for preemployment screening purposes has been seriously questioned $(7,8,26)$. On the other hand it may be argued that an asthma prevalence of $12 \%$ is high indeed and that the theoretical reduction achieved by the exclusion of atopics would not be negligible. Data on the incidence and prognosis of laboratory animalinduced allergy are needed; these can only be obtained from longitudinal studies.

Man-made chemicals are even less predictable than proteins. During the late 1960s and 1970s the detergent industry had a specific problem with Bacillus subtilis enzymes, which caused IgE-mediated allergy. Longitudinal studies using skin tests revealed that atopy predisposed for $B$ subtilis sensitization. Although the skin reactivity to the proteolytic enzymes appeared unaccompanied by clinical symptoms, atopics were excluded at the preemployment examinations (22). Atopy also seems to predispose strongly for sensitization to platinum salts (Newman Taylor, personal communication).

The opposite applies to exposure to diisocyanates. The risk of acquiring asthma from this group of chemicals is at least as great for nonatopics as for atopics (29). The distribution of atopy among 92 cases of diisocyanate asthma, all of which were confirmed by bronchial provocation tests at the Institute of Occupational Health in Helsinki, is shown in table 3. Atopy did not seem to be associated with any propensity to contract disease. The same was true for 12 cases of formaldehyde asthma (28). These findings are not surprising, as there is no reason to believe that a reaction to formaldehyde would be IgE-mediated; likewise IgE rarely seems to be of importance in isocyanate-induced asthma. Also in the sensitization of the respiratory tract by phthalic anhydride, atopy appears to be of little relevance (table 3 ). Weeding out symptomless atopics from such work would be a form of malpractice.

\section{Prognosis of sensitized individuals}

The prognosis of occupational allergic disease is an important aspect when the risks involved in sensitization are weighed. In general such knowledge is lacking. There are reports showing that asthma caused by red cedar (4) and colophony (3) may persist long after the subject is no longer exposed.

A study on toluene diisocyanate workers indicated that symptoms may continue for years and even de-
Table 1. Prevalence of laboratory animal allergy (LAA) and asthma.

\begin{tabular}{lccc}
\hline & & \multicolumn{2}{c}{ Prevalence } \\
\cline { 3 - 4 } Reference & $\begin{array}{c}\text { Population } \\
\text { studied (N) }\end{array}$ & $\begin{array}{c}\text { LAA } \\
(\%)\end{array}$ & $\begin{array}{c}\text { Asthma } \\
(\%)\end{array}$ \\
\hline Taylor et al (34) & 474 & 23 & 9 \\
Gross (16) & 399 & 15 & 7.5 \\
Cockcroft et al (7) & 179 & 27 & 12 \\
Davies \& McArdle (10) & 585 & 20 & 3 \\
Newman Taylor et al (27) & 144 & 27 & 11 \\
Slovak \& Hill (33) & 146 & 30 & 10 \\
Davies et al (11) & 148 & $\mathbf{1 5}$ & 2 \\
\hline
\end{tabular}

a One-year follow-up.

Table 2. Distribution of animal-related allergy and asthma in relation to atopy (7).

\begin{tabular}{lccccc}
\hline & \multicolumn{2}{c}{ Atopics } & & \multicolumn{2}{c}{ Nonatopics } \\
\cline { 2 - 3 } & Number Percent & & Number Percent \\
\cline { 2 - 3 } $\begin{array}{l}\text { All animal-related } \\
\text { symptoms }\end{array}$ & 21 & 30 & & 28 & 26 \\
$\begin{array}{l}\text { Asthma from animal } \\
\text { contact }\end{array}$ & 14 & 20 & 7 & 6 \\
$\begin{array}{l}\text { No symptoms related } \\
\text { to animals }\end{array}$ & 49 & 70 & & 81 & 74 \\
\hline Total & 70 & 100 & 109 & 100 \\
\hline
\end{tabular}

Table 3. Atopy among 92 cases of diisocyanate asthma, 12 cases of asthma caused by formaldehyde, and 11 by phthalic anhydride. All cases were confirmed with bronchial provocation tests.

\begin{tabular}{lccc}
\hline Agent & Atopic & Nonatopic & All \\
\hline Diisocyanate $^{\mathrm{a}}$ & 22 & 70 & 92 \\
Formaldehyde & 2 & 10 & 12 \\
Phthalic anhydride & 2 & 9 & 11 \\
\hline
\end{tabular}

a Toluene diisocynate, 4,4-diphenylmethane diisocyanate, or 1,6-hexamethylene diisocyanate.

teriorate after the exposure is discontinued (1). This is in accordance with the experience gained on isocyanate asthma at the Institute of Occupational Health, in Helsinki. A self-administered questionnaire mailed to 92 confirmed cases, 84 of whom responded, revealed that 8 of the 14 who had continued in isocyanate-exposed work believed that their health had deteriorated, whereas only two felt better. Of the 70 workers who had no further exposure, 50 reported that the symptoms $0.5-5$ years later had continued without clear improvement, and another 13 felt their health had deteriorated. Only seven reported amelioration. Although the results express subjective assessments, they leave the impression that isocyanates may trigger an asthma which develops into a nonspecifically reacting disease.

The study by Järvinen and co-workers (21) on bakers suggested a more optimistic prognosis; many workers with asthma managed to continue with mild or no symtoms when transferred to worksites with 
less exposure to flour dust. Two prospective studies on bakers have reported a so far unexplained phenomenon. Workers who turned skin positive to flour extracts during the first year of exposure sometimes became negative during subsequent years $(14,19$, 20). Only prospective studies will be able to tell the predictive value of skin positivity in relation to clinical disease.

\section{Mechanisms of disease}

There are two principal explanations for the fact that the occurrence of work-related symptoms can be little reduced by the exclusion of atopics. First, while atopy is, by definition, confined to the production of IgE antibody, this class of antibody is not limited to atopics. It is a normal class of antibody, and there is ample evidence showing that nonatopics may develop an IgE-mediated allergy, provided that the intensity of exposure is strong enough and/or that the sensitizing properties of the offending agent are potent enough. The second explanation is that there are several other mechanisms for the disease.

With respect to occupational asthma, the direct action on vagal receptors in the trachea and bronchi obviously plays an important role in the pathogenesis. Asthma can also be induced by a variety of factors capable of activating the mast cell and the basophil (23). Such factors include antigen-antibody reactions other than IgE, eg, short-term sensitizing antibody (STS-A) and other IgG reactions, chemical and mechanical factors, interference with the intracellular c-AMP/c-GMP balance, etc. It seems likely that the role of mechanisms other than IgE-mediated ones are particularly important in the induction of occupational asthma, as the exposure to many potent agents may be several times higher in occupational environments than elsewhere.

\section{Discussion}

Atopy is a common characteristic. Any decision taken on atopy per se will affect about one-third of job applicants. Such far-reaching decisions should be based on indisputable evidence that atopics are at a greater risk; the main interest of occupational health officers should be to protect workers from disease, not work.

While some evidence exists from certain work environments, such as the earlier processes of the detergent industry and work with platinum salts, that atopy is relevant as a risk predictor, such knowledge is lacking for most occupations. Other occupations, including all those associated with exposure to diisocyanates and formaldehyde, show no indication of any increased risk for atopics. Some data on positive skin tests with common allergens and atopic family history among bakers can be construed to suggest an increased risk of sensitization to flour $(21,35)$. Several studies show that the prevalence of asthma among laboratory animal handlers is high and that the risk of progression into asthma is clearly increased among atopics. Although screening for atopy would reduce the cases of asthma in this particular work, present data on the predictive value of atopy suggest that the characteristic is rarely discriminatory enough to require exclusion from a certain environment. Prospective studies from various occupational environments are needed before further recommendations can be made. However, atopy may well serve as a means of identifying individuals who, because of the marginally increased risk, should be subjected to more careful medical supervision, including information about risks and means of protection.

Individuals displaying symptoms in a preemployment examination merit a separate discussion. There are other and more clear-cut aspects guiding decisions regarding such workers. Thus subjects with dermatitis are well known to be unsuitable in a variety of environments such as kitchen work, animal handling, hairdressing, etc. The nonspecific bronchial hyperreactivity of asthmatics render this group of applicants unsuitable for any dusty work or any environment associated with irritants. In general, symptomatic workers are unsuitable for work in environments where their symptoms will deteriorate. Such decisions include medicolegal aspects; there may be difficulties in distinguishing between aggravation of a preexisting disease and disease caused occupationally. However, this difficulty applies to workers regardless of the mechanism of disease, be it extrinsic or intrinsic asthma or atopic or nonatopic dermatitis. It is not tied to atopy. As regards symptomless subjects who claim previous experiences of atopic symptoms during childhood and adolescence, but who have been asymptomatic ever since, the decisions may be less self-evident. The present literature does not give much guidance with respect to the risk of relapse of former atopic symptoms in various occupations. Until there is evidence of a clearly increased risk it seems reasonable not to put undue weight on past episodes, or a family history of atopy.

One is well advised to keep in mind that workrelated symptoms cannot be eradicated by the exclusion of atopics. The reasons are obvious: (i) nonatopics are capable of producing IgE when exposed to strong allergens or haptens, (ii) the mechanisms of disease are multiple, and (iii) only some of these mechanisms are linked to IgE and atopy. Moreover any increased risk should be carefully weighed against the untowarded implications involved in unemployment. When there is a clear risk, screening policies ought to be applied during vocational guidance or, at the latest, when a prospective student applies to a vocational school. Finally, screening for susceptibility may be a double-edged practice. There is an inherent risk of forgetting about hygienic and 
technical measures to reduce exposure, which eventually is the only efficient way to achieve a substantial reduction of work-related allergy.

\section{References}

1. Adams WGF. Long-term effects on the health of men engaged in the manufacture of toluene di-isocynate. $\mathrm{Br}$ $J$ Ind Med 32 (1975) 72-78.

2. Barbee RA, Lebowitz MD, Thompson HC, Burrows B. Immediate skin test reactivity in a general population sample. Ann Intern Med 84 (1976) 129-133.

3. Burge PS, Harries MG, O'Brien IM, Pepys J. Respiratory disease in workers exposed to solder flux fumes containing colophony (pine resin). Clin Allergy 8 (1978) 1-14.

4. Chan-Yeung M. Fate of occupational asthma: A followup of patients with occupational asthma due to western red cedar (Thuja Plicata). Am Rev Respir Dis 116 (1977) 1023-1029.

5. Coca AF, Cooke RA. On the classification of the phenomena of hypersensitiveness. J Immunol 8 (1923) $163-182$.

6. Coca AE, Grove E. Studies in hypersensitiveness: XIII A study of the atopic reagins. J Immunol 10 (1925) 445-464.

7. Cockcroft A, Edwards J, McCarthy P, Andersson N. Allergy in laboratory animal workers. Lancet 1 (1981) 827-830.

8. Cockcroft A, Edwards J, McCarthy P. The role of preemployment allergy screening in animal work. Eur $\mathbf{J}$ Respir Dis 62 (1981): suppl 113, 42-43.

9. Curran WS, Goldman H. The incidence of immediate reacting allergen skin tests in the normal adult population. Ann Intern Med 55 (1961) 777-783.

10. Davies GE, McCardle LA. Allergy to laboratory animals: A survey by questionnaire. Int Arch Allergy Appl Immunol 64 (1981) 302-307.

11. Davies GE, Thompson AV, Niewola Z, Burrows GE, Teasdale EL, Bird DJ, Phillips DA. Allergy to laboratory animals: A retrospective and a prospective study. Br J Ind Med 40 (1983) 442-449.

12. D'Souza MD, Davies RJ. The distribution of allergic disorders and atopy in the community and their relationship to total levels of serum IgE antibody. Am Rev Respir Dis 115 (1977) 211

13. Freeman GL, Johnson S. Allergic diseases in adolescents: I Description of survey, prevalence of allergy. Am J Dis Child 107 (1964) 549-559.

14. Gadborg E. Om Melallergi. Christtreus Bogtryckeri, København 1956. (Doctoral dissertation).

15. Godfrey RC, Griffiths M. The prevalence of immediate positive skin tests to Dermatophagoides pteronyssinus and grass pollen in school children. Clin Allergy 6 (1976) 79-82.

16. Gross NJ. Allergy to laboratory animals: Epidemiologic clinical and physiologic aspects, and a trial of cromolyn in its management. J Allergy Clin Immunol 66 (1980) $158-165$.

17. Haahtela T. Allergy in young people: An epidemiological study of immediate skin test reactivity and allergic disorders among adolescents and conscripts in southeast Finland. University of Helsinki, Helsinki 1980. (Doctoral dissertation).
18. Hagy WG, Settipane GA. Bronchial asthma, allergic rhinitis and allergy among college students. J Allergy 6 (1969) 79.

19. Herxheimer H. Die Entwicklung von Mehlempfindlichkeit der Haut bei Bäckerlehrlingen und Bäckern. Klin Wochenchr 45 (1967) 481-484.

20. Herxheimer H. The skin sensitivity to flour of bakers' apprentices. Acta Allergol 28 (1973) 42-49.

21. Järvinen KAJ, Pirilä V, Björksten F, Keskinen $H$, Lehtinen M, Stubb S. Unsuitability of bakery work for a person with atopy: A study of 234 bakery workers. Ann Allergy 42 (1979) 192-195.

22. Juniper CP, How MJ, Goodwin BFJ, Kinshott AK. Bacillus subtilis enzymes: A 7-year clinical, epidemiological and immunological study of an industrial allergen. J Soc Occup Med 27 (1977) 3-12.

23. Kay AB. Basic mechanisms in allergic asthma. Eur $\mathbf{J}$ Respir Dis 63 (1982): suppl 122, 9-16.

24. Lincoln TA, Bolton NE, Garrett AS. Occupational allergy to animal dander and sera. J Occup Med 16 (1974) 465-469.

25. Lubs ML. Allergy in 7000 twin pairs. Acta Allergol 26 (1971) 249-285.

26. Newman Taylor AJ. Laboratory animal allergy. In: Kerr JW, Ganderton MA, ed. XI international congress of allergology \& clinical immunology, London 17-22 October 1982. MacMillan Press Ltd, London 1983, pp 445-448.

27. Newman Taylor AJ, Myers JR, Longbottom JL, Spackman D, Slovak AJM. Immunological differences between asthma and other allergic reactions in laboratory animal workers. Thorax 36 (1981) 229.

28. Nordman H, Keskinen H, Tuppurainen M. Formaldehyde asthma - Rare or overlooked? J Allergy Clin Immunol (in press).

29. O'Brien IM, Newman Taylor AJ, Burge PS, Harries MG, Fawcett IW, Pepys J. Toluene di-isocyanateinduced asthma: II Inhalation challenge tests and bronchial reactivity studies. Clin Allergy 9 (1979) 7-15.

30. Patterson R. Investigation of spontaneous hypersensitivity of the dog. J Allergy 31 (1969) 351-354.

31. Pepys J. Atopy. In: Gell PHC, Coombs RRA, Lachmann PJ, ed. Clinical aspects of allergy. Third edition. Blackwell Scientific Publications, Oxford 1975, pp $877-902$.

32. Ramazzini B. De morbis artificum diatriba: Padua 1700 Ex typograhica Caroli Calumbi, Rome 1700.

33. Slovak AJM, Hill RN. Laboratory animal allergy: A clinical survey of an exposed population. $\mathrm{Br} \mathrm{J}$ Ind Med 38 (1981) 38-41.

34. Taylor G, Davies GE, Altounyoan REC. Allergic reactions to laboratory animals. Nature 60 (1976) $230-232$.

35. Thiel H. Baker's asthma: Epidemiological and clinical findings - Need for prospective studies. In: Kerr JW, Ganderton MA, ed. XI international congress of allergology \& clinical immunology, London 17-22 October 1982. MacMillan Press Ltd, Dondon 1983, pp $429-433$.

36. Vaughan WT. Practice of allergy. Third edition. 1954, p 55.

37. Wilbur RD, Ward GW. Immunologic studies in a case of baker's asthma. J Allergy 58 (1976) 366-372.

38. Woolcock AJ, Colman MH, Jones MW. Atopy and bronchial reactivity in Australian and Melanesian populations. Clin Allergy 8 (1978) 155. 